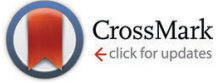

Cite this: Phys. Chem. Chem. Phys., $2016,18,31268$

Received 18th July 2016, Accepted 24th October 2016

DOI: $10.1039 / c 6 c p 04984 b$

www.rsc.org/pccp

\section{Adsorption and diffusion of Li with S on pristine and defected graphene $\dagger$}

\author{
Zhicong Liang, ${ }^{a}$ Xiaofeng Fan, ${ }^{{ }^{a}}$ David J. Singh ${ }^{\mathrm{ab}}$ and W. T. Zheng ${ }^{{ }^{\mathrm{ac}}}$
}

$\mathrm{Li}-\mathrm{S}$ batteries are the promising high energy density alternative to current rechargeable battery technologies, particularly since it has been shown that the use of graphene, nanotubes and other nanostructured carbons in the cathode can improve the cyclability. We explore the microscopic interactions between $\mathrm{Li}_{n} \mathrm{~S}$ and graphene and diffusion of $\mathrm{Li}$ ions through pristine and defected graphene in the presence of $\mathrm{S}$ using first-principles methods. The introduction of Li weakens the interaction of atomic S with graphene, increasing the height of adsorbed $\mathrm{S}$ and leading to the formation of $\mathrm{Li}_{n} \mathrm{~S}$ clusters. These $\mathrm{Li}_{n} \mathrm{~S}$ clusters are adsorbed accompanied by charge transfer to the graphene. We find that double vacancies in the graphene are sufficient to allow $\mathrm{Li}$ ions to pass through the graphene plane. This is impeded in the presence of $S$ due to the binding of $\mathrm{Li}$ to $\mathrm{Li}_{n} \mathrm{~S}$ clusters, but still can happen for larger clusters. The electronic properties confirm the excellent conductivity of pristine and defected graphene cathodes in contact with $\mathrm{Li}_{n} \mathrm{~S}$ clusters.

\section{Introduction}

Energy storage in the form of rechargeable batteries is central to many modern technologies. However, current Li-ion batteries have insufficient capacities to meet the demands of key markets. ${ }^{1-7}$ The currently used transition metal oxide based cathodes have theoretical specific capacities of $\sim 300 \mathrm{~mA} \mathrm{~h} \mathrm{~g}^{-1}$, which is very limiting, for example in vehicle electrification. ${ }^{7-9}$ Recently, Li-S batteries have attracted much more attention due to the large specific capacity $\left(1673 \mathrm{~mA} \mathrm{~h} \mathrm{g^{-1 }}\right)$ of sulfur as the cathode. Based on the reaction $\mathrm{S}_{8}+16 \mathrm{Li}^{+}+16 \mathrm{e}^{-} \leftrightarrow 8 \mathrm{Li}_{2} \mathrm{~S}$ with an average working voltage at around $2.1 \mathrm{~V}\left(\mathrm{~V}\right.$ vs. $\left.\mathrm{Li} / \mathrm{Li}^{+}\right), \mathrm{Li}-\mathrm{S}$ batteries with a lithium anode can offer very high theoretical energy densities (gravimetric energy density of $\sim 2600 \mathrm{~W} \mathrm{~h} \mathrm{~kg}^{-1}$ and volumetric energy density of $\left.2500 \mathrm{~W} \mathrm{~h} \mathrm{~L}^{-1}\right) \cdot{ }^{10-18}$ In addition, sulfur is sufficient in the earth, cheap and environmentally friendly. ${ }^{5,19,20}$ However, the shortcomings also are obvious, including the low electrical conductivity of sulfur $S_{8}\left(5 \times 10^{-30} \mathrm{~S} \mathrm{~cm}^{-1}\right.$ at $25{ }^{\circ} \mathrm{C}$ ), solution phase reduction of long chain polysulfide to

\footnotetext{
${ }^{a}$ College of Materials Science and Engineering, Key Laboratory of Automobile Materials of MOE, Jilin University, Changchun 130012, China.

E-mail: xffan@jlu.edu.cn,wtzheng@jlu.edu.cn

${ }^{b}$ Department of Physics and Astronomy, University of Missouri, Columbia, Missouri 65211-7010, USA

${ }^{c}$ State Key Laboratory of Automotive Simulation and Control, Jilin University, Changchun 130012, China

$\dagger$ Electronic supplementary information (ESI) available: Possible configurations of $\mathrm{S}$ and $\mathrm{Li}_{n} \mathrm{~S}(n=1,2)$ on pristine graphene and double-vacancy graphene with the important parameters calculated in Fig. S1-S10 and Tables S1-S10. The band structure, DOS and PDOS of S-adsorbed graphene in Fig. S11 and DOS and PDOS of two-vacancy graphene with the adsorption of LiS in Fig. S12. See DOI: 10.1039/ c6ср04984b
}

short-chain polysulfide, and the large volume $(\sim 76 \%)$ and morphology changes of sulfur electrodes during the dischargecharge process. ${ }^{21-27}$ Short-chain polysulfides, such as $\mathrm{Li}_{2} \mathrm{~S}_{4-8}$, are easily dissolved in the electrolyte solution, and this results in the mass loss of active sulfur. ${ }^{28}$ These shortcomings limit the cycle life leading to a series of issues including the capacity fade, low Coulombic efficiency during cycling, lithium metal corrosion, and dendrite formation. ${ }^{13,16}$ The significant challenges to develop these batteries remain, particularly because of the need to contain sulfur in the cathode due to the large volume changes upon charging and discharging and the need to obtain conduction due to the insulating nature of $\mathrm{S}$.

In order to overcome these obstacles, various approaches have been proposed to raise the actual capacity and the cycle stability of the sulfur cathode. In order to prevent the dissolution of lithium polysulfide, a big tide in the research is the confining of sulfur in nano-carbons. ${ }^{14,20,28,29}$ Commonly, a composite cathode consisting of sulfur in various carbon matrices, such as meso-/micro-porous carbon, graphene or graphene oxide, carbon nanotubes, carbon fibers, hollow carbon microspheres, is used. ${ }^{30-32}$ It is generally understood that the nano-structured carbon performs two functions: (1) its sorption properties provide the close contact between the sulfur and a conductive network and (2) it encapsulates or otherwise impedes the diffusion of polysulfides into the electrolyte. ${ }^{33}$ However, the microscopic mechanisms, which will be important for optimizing the electrode structures, are poorly understood at present. Interestingly, there are recent reports that graphene-based sulfur cathodes may be used in Li-S batteries and the graphene-encapsulation layers can substantially improve the cycling life. , $13,15,18,31,34-38$ The binding between lithium polysulfide and doped graphene (such as the 
B and $\mathrm{N}$ co-doping) has been reported theoretically. ${ }^{39}$ In addition, to provide a practical cathode material, this gives us a model system for understanding the detailed microscopic mechanisms operative in nano-carbon-sulfur cathodes.

Here we focus on the interaction between $\mathrm{Li}_{n} \mathrm{~S}$ clusters and graphene and the diffusion of Li through graphene in the presence of S. Specifically we analyze the adsorption of Li including the formation of $\mathrm{Li}_{n} \mathrm{~S}$ clusters on pristine and vacancy-defected graphene. Then the desorption of $S$ from graphene and at the edge of double-vacancy both by itself and in clusters with $\mathrm{Li}$ is explored. We find that Li greatly assists the desorption of S via the formation of $\mathrm{Li}_{n} \mathrm{~S}$ clusters, the same process works against the diffusion of $\mathrm{Li}$ through the graphene plane. However, this inhibition is only present for the small size of clusters, and $\mathrm{Li}$ from larger size $\mathrm{Li}_{n} \mathrm{~S}$ can readily diffuse through divacancy pores in graphene. The results can be understood in terms of the incremental binding of $\mathrm{Li}$ to $\mathrm{Li}_{n} \mathrm{~S}$ clusters and the charge transfer between $\mathrm{Li}, \mathrm{S}, \mathrm{Li}_{n} \mathrm{~S}$ clusters and graphene. Additionally, we present the analysis of electronic structures, which confirms the conductivity of the carbon system in the presence of $\mathrm{Li}$ and $\mathrm{S}$.

\section{Computational methods}

Our calculations have been carried out based on density functional theory employing the projector augmented wave potentials as implemented in the VASP code. ${ }^{40-42}$ We used the generalized gradient approximation (GGA) with the parameterization of Perdew-Burke-Ernzerhof (PBE) ${ }^{43}$ and added van der Waals (vdW) corrections for the adsorption on the surface. The $k$-point sampling of the Brillouin zone and the plane-wave basis sets were chosen to obtain the total energy convergence at the $1 \mathrm{meV}$ per atom level. This corresponds to a kinetic energy cutoff of $450 \mathrm{eV}$ for the plane wave expansion. We did the spin-polarized calculations to include the moments on isolated $\mathrm{Li}$ atoms and defective graphene. The effect of the dispersion interaction is included by the empirical correction scheme of Grimme (DFT+D/PBE). ${ }^{44}$ This approach has been successful in describing graphene-based structures. $^{45,46}$

The lattice constant of graphene is calculated to be $2.468 \AA$, which is basically identical to that from previous work, ${ }^{47}$ and is a little larger than the experimental value of $2.46 \AA$. For graphene with a double-vacancy, the change of lattice parameters in a $4 \times 4$ cell is found to be small and can be ignored. To treat the adsorption of Li and S, we used a supercell method, specifically a $4 \times 4$ supercell with a vacuum layer of $18 \AA$. The Brillouin zone was sampled with the $\Gamma$-centered $12 \times 12 \times 1$ grid of $k$-points. We note that the adatom-graphene system lacks inversion symmetry and therefore requires the use of a large vacuum to reduce the spurious effects due to the dipole interactions, ${ }^{48}$ which is the reason for the large vacuum layer in our calculations. The in-plane supercell lattice constant is $9.87 \AA$, which is also the distance between the neighboring adatoms (or clusters).

\section{Results and discussion}

\section{Adsorption of Li with $S$ on pristine and double-vacancy graphene}

We start with the analysis for the $S_{2}$ molecule. We obtain an S-S bond length of $1.90 \AA$, which is similar to the experimental value of $1.889 \AA{ }^{49,50}$ With the consideration of the different configurations of $S_{2}$ and $S_{8}$ adsorbed on graphene (see Fig. S1, $\mathrm{S} 2$, Tables $\mathrm{S} 1$ and $\mathrm{S} 2$ of ESI $\dagger$ ), it is found the adsorption energy of poly-sulfur on pristine graphene is small. For example, with the consideration of the vdW effect, the adsorption energies of $\mathrm{S}_{8}$ and $\mathrm{S}_{2}$ on graphene are $-0.088 \mathrm{eV}$ per S-atom and $-0.094 \mathrm{eV}$ per S-atom, respectively. The interaction between poly-sulfur and graphene is mainly by the dispersion force. With the increase of the atom number in poly-sulfur, the interaction between poly-sulfur, such as $\mathrm{S}_{8}$, and graphene becomes stronger. On the other hand, the interaction between single-atom $S$ and graphene is considerable due to charge transfer. The most stable site on pristine graphene among the possible sites, such as hollow, bridge and top sites, is the bridge site between two carbon atoms (in Fig. 1A) with an adsorption energy of $-0.995 \mathrm{eV}$ per S-atom (see Fig. S3 and Table S3 of ESI $\dagger$ ).

With double-vacancy on graphene by which it is expected that Li can diffuse from one side of graphene to the other side, the interaction between poly-sulfur and defected graphene isn't strengthened obviously, for example, the adsorption energies of $\mathrm{S}_{8}$ and $\mathrm{S}_{2}$ are $-0.092 \mathrm{eV}$ per $\mathrm{S}$-atom and $-0.091 \mathrm{eV}$ per $\mathrm{S}$-atom (see Fig. S4, S5, Tables S4 and S5 of ESI $\dagger$ ). The localized states near the Fermi level introduced by double-vacancy can't enhance the interaction between poly-sulfur and graphene obviously. While the interaction between single-atom $\mathrm{S}$ and defected graphene becomes much stronger due to the formation of a chemical bond. The most stable one on double-vacancy graphene is the edge of the
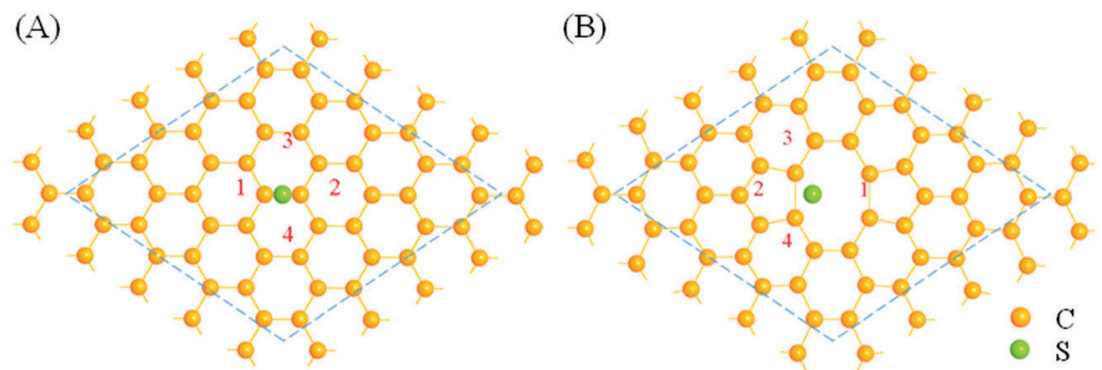

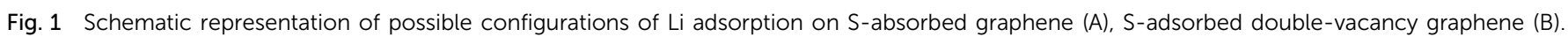
Note the numbers in the figures represent the number and corresponding possible locations of Li atoms. 
vacancy with a 5-ring (in Fig. 1B) with an adsorption energy of $-4.22 \mathrm{eV}$ per S-atom. This is based on the results for the different possible adsorption sites (see Fig. S6 and Table S6 of ESI†).

For graphene as the encapsulation layer, it is expected to prevent the dissolution of lithium polysulfide into electrolytes, while Li-ions can easily diffuse from the one side of the encapsulation layer (with lithium polysulfide) to the other side (into the electrolytes). It is well known that the chain of polysulfide will become shorter upon reaction with $\mathrm{Li}$ (from $\mathrm{S}_{8}$ to $\mathrm{Li}_{2} \mathrm{~S}$ ). Therefore, the $\mathrm{Li}_{n} \mathrm{~S}$ cluster will be possible stay near the surface of graphene, since the interaction between polysulfide and graphene is weaker. We next consider Li with $\mathrm{S}$ on pristine and double-vacancy graphene.

Possible adsorption configurations were investigated by relaxing the adatom $\mathrm{Li}$ around the $\mathrm{S}$ atom on S-adsorbed graphene with the consideration of symmetry (see Fig. S7-S10 and Tables S7-S10 of ESI $\dagger$ ). ${ }^{51}$ With these results, the possible adsorption sites of $\mathrm{Li}_{n}(n=1,2,3$ and 4 ) around $\mathrm{S}$ adsorbed on graphene were investigated, as depicted in Fig. 1A and B. Taking $\mathrm{Li}_{n} \mathrm{~S}$ as a molecular cluster adsorbed on graphene, the adsorption energy can be defined by the formula

$$
E_{\mathrm{ad}}=E_{\mathrm{ad}-\mathrm{g}}-E_{\mathrm{g}}-E_{\mathrm{ad}},
$$

where $E_{\mathrm{ad}-\mathrm{g}}, E_{\mathrm{g}}$ and $E_{\mathrm{ad}}$ are the total energies of the system of $\mathrm{Li}_{n} \mathrm{~S}$-graphene, isolated graphene and isolated adsorbate $\mathrm{Li}_{n} \mathrm{~S}$. This energy quantifies the interaction between graphene and adsorbate $\mathrm{Li}_{n} \mathrm{~S}$. Turning to the $\mathrm{Li}$, the energy of $\mathrm{Li}_{n}$ adsorption per atom (cohesive energy) can be obtained as

$$
\Delta E_{\mathrm{c}}=\left(E_{\mathrm{ad}-\mathrm{g}}-E_{\mathrm{g}-\mathrm{S}}-n E_{\mathrm{Li}}\right) / n
$$

where $E_{\mathrm{ad}-\mathrm{g}}, E_{\mathrm{g}-\mathrm{S}}$ and $E_{\mathrm{Li}}$ are the total energies of the system of $\mathrm{Li}_{n} \mathrm{~S}$-graphene, isolated $\mathrm{S}$-adsorbed graphene and isolated $\mathrm{Li}$ atoms. This energy shows the influence of $\mathrm{Li}$ on the adsorption of $\mathrm{S}$ on graphene. The geometric structure and adsorption energy were obtained after all the atoms in the models were fully relaxed. Furthermore, the lattice parameters were additionally relaxed with double-vacancy in order to account for distortions that could occur. In this case, the in-plane supercell lattice constant is reduced to about $9.67 \AA$.

The results of calculations are listed in Table 1 . It is found that the adsorption energy of $\mathrm{Li}_{n} \mathrm{~S}$ clusters gradually increases as the number of $\mathrm{Li}$ atoms increases. By comparing the distance between graphene and S $\left(d_{\mathrm{S}-\mathrm{C}}\right)$ in S-graphene (Table S3 of ESI $\dagger$ ) and LiS-graphene (Table 1), the height of the $S$ atom is increased due to the adsorption of $\mathrm{Li}$ on graphene. Li results in the weakening of bonds between $S$ and graphene. By the value of $d_{\mathrm{Li}-\mathrm{C}}, d_{\mathrm{S}-\mathrm{C}}$, and $\theta_{\mathrm{C}-\mathrm{S}-\mathrm{C}}$, graphene prefers $\mathrm{Li}$ atoms to $\mathrm{S}$ atoms. The value of $d_{\mathrm{S}-\mathrm{Li}}$ (about 2.2 $\AA$ ) in $\mathrm{Li}_{n} \mathrm{~S}$ clusters implies the direct interaction between $\mathrm{Li}$ and $\mathrm{S}$. Therefore, $\mathrm{Li}-\mathrm{S}$ clusters are formed on graphene. Based on the cohesive energy (Table 1) defined in formula (2), the interaction between Li and S-graphene is stronger than the binding between $\mathrm{Li}$ atoms in bulk Li where the binding energy is about $1.61 \mathrm{eV}$ per Li.

For the defected graphene, the interaction between $\mathrm{S}$ and the edge of double vacancy is very strong. Single $\mathrm{Li}$ atoms don't raise $\mathrm{S}$ atoms adsorbed on double-vacancy graphene. The distance
Table 1 Average distance between $\mathrm{S}$ and $\mathrm{Li}\left(d_{\mathrm{S}-\mathrm{L}}\right)$, average distance between $\mathrm{Li}$ and near-neighbor $\mathrm{C}\left(d_{\mathrm{Li}-\mathrm{C}}\right)$, distance between $\mathrm{S}$ and the nearest-neighbor $C\left(d_{s_{-}-}\right)$, angle between $S$ and the near-neighbor $C\left(\theta_{C-S_{-}}\right)$, adsorption energy $\left(E_{\mathrm{ad}}\right)$ and cohesive energy $\left(\Delta E_{\mathrm{c}}\right)$ for the adsorption of $\mathrm{Li}_{\mathrm{n}} \mathrm{S}$ cluster on pristine graphene and double-vacancy graphene

\begin{tabular}{llllllll}
\hline & $\mathrm{N}$ & $d_{\mathrm{S}-\mathrm{Li}}$ & $d_{\mathrm{Li}-\mathrm{C}}$ & $d_{\mathrm{S}-\mathrm{C}}$ & $\theta_{\mathrm{C}-\mathrm{S}-\mathrm{C}}$ & $E_{\mathrm{ad}}(\mathrm{eV})$ & $\Delta E_{\mathrm{c}}(\mathrm{eV})$ \\
\hline Graphene & 1 & 2.23 & 2.37 & 3.67 & 21.6 & -0.813 & -3.136 \\
& 2 & 2.20 & 2.35 & 3.51 & 23.4 & -1.086 & -3.326 \\
& 3 & 2.20 & 2.35 & 3.57 & 23.1 & -2.800 & -3.340 \\
& 4 & 2.32 & 2.30 & 3.47 & 23.8 & -4.289 & -3.065 \\
Graphene-dv & 1 & 2.56 & 2.10 & 1.75 & 94.0 & -3.212 & -2.307 \\
& 2 & 2.17 & 2.30 & 2.94 & 34.5 & -1.501 & -1.919 \\
& 3 & 2.21 & 2.37 & 3.67 & 26.4 & -3.622 & -2.538 \\
& 4 & 2.33 & 2.31 & 3.47 & 27.1 & -6.100 & -2.710
\end{tabular}

between $S$ and graphene is about $1.75 \AA$, as shown in Fig. S9 and Table S9 of ESI. $\dagger$ Interestingly, on the other hand, as few as two $\mathrm{Li}$ atoms can result in the lifting of $\mathrm{S}$ on double-vacancy graphene in Table 1. With the introduction of the second $\mathrm{Li}$, a $\mathrm{Li}_{2} \mathrm{~S}$ cluster is formed with the breaking of $\mathrm{S}-\mathrm{C}$ bonds. Therefore, the adsorption energy of $\mathrm{Li}_{2} \mathrm{~S}$ is significantly lower than that of LiS on double-vacancy graphene.

\section{Dynamics of S-desorption under the influence of adsorbed $\mathrm{Li}$ and diffusion of $\mathrm{Li}$ with S-adsorption on double-vacancy graphene}

One use of graphene in the cathode is as an encapsulation layer to enhance the electronic conduction of the sulfur cathode while preventing the dissolution of polysulfide into the electrolyte. There are important issues about the dynamical processes of S-desorption and Li-diffusion through the graphene plane. As above, $\mathrm{Li}$ atoms can make the adsorption of single-S on pristine graphene unstable. As shown in Fig. 2B, there is a dynamical barrier of approximately $0.34 \mathrm{eV}$ in the desorption process. Interestingly, with the introduction of two Li atoms, the barrier in the process is zero (Fig. 2). This means that $\mathrm{Li}_{n} \mathrm{~S}$ clusters will be formed easily when $\mathrm{Li}$ ions diffuse from the electrolyte into the cathode upon the discharge of the battery, in the case of pristine graphene as an encapsulation layer.

For defected graphene, such as double-vacancy containing graphene, the binding of $\mathrm{S}$ at the edge of the defect is stronger. As shown in Fig. 3, when a single Li is introduced, the adsorption of $S$ at the edge of vacancy is still stable. With the introduction of two $\mathrm{Li}$ atoms, the adsorption of $\mathrm{S}$ becomes unstable. However, there is a dynamical barrier of about $0.80 \mathrm{eV}$ for the S-desorption and formation of $\mathrm{Li}_{2} \mathrm{~S}$. With three $\mathrm{Li}$ atoms, the process of desorption has no dynamical barrier and $\mathrm{Li}_{3} \mathrm{~S}$ clusters are then readily formed.

The diffusion of $\mathrm{Li}$ from $\mathrm{Li}_{n} \mathrm{~S}$ by going through the graphene encapsulation layer is important. Prior work ${ }^{52}$ shows that $\mathrm{Li}$ cannot diffuse in the direction perpendicular to the graphene sheet for pristine graphene or/and graphene with single vacancies. The smallest pore through which Li can pass through graphene is the double-vacancy. Here the diffusion of $\mathrm{Li}$ in the $\mathrm{Li}_{n} \mathrm{~S}$ cluster through the graphene plane with double-vacancies is analyzed in Fig. 4. Two models, specifically $\mathrm{Li}_{2} \mathrm{~S}$ and $\mathrm{Li}_{3} \mathrm{~S}$, were 

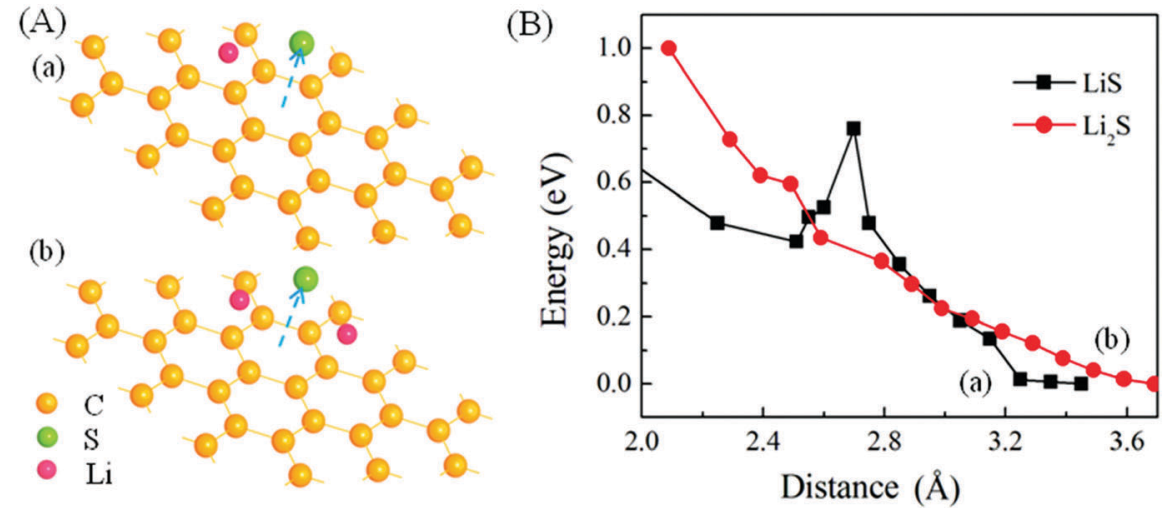

Fig. 2 Schematic representations of the dynamical process of S-desorption on graphene with the single-Li adsorption (a, LiS cluster) and double Li ion adsorption $\left(b, \mathrm{Li}_{2} \mathrm{~S}\right)(\mathrm{A})$, and the potential-energy curves of $\mathrm{S}$-desorption in both cases $(\mathrm{B})$. Note that the direction of $\mathrm{S}$ diffusion is perpendicular to the graphene sheet and the zero of energy is cited at the position where $S$ is far way from the graphene plane.

(A)

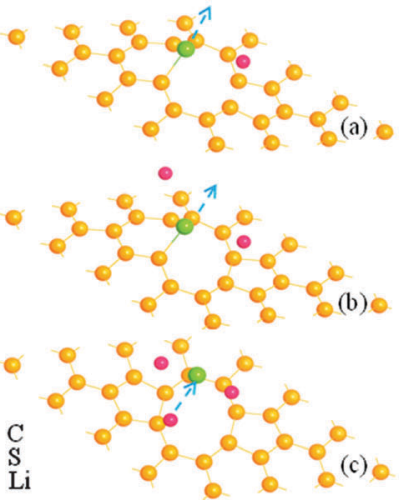

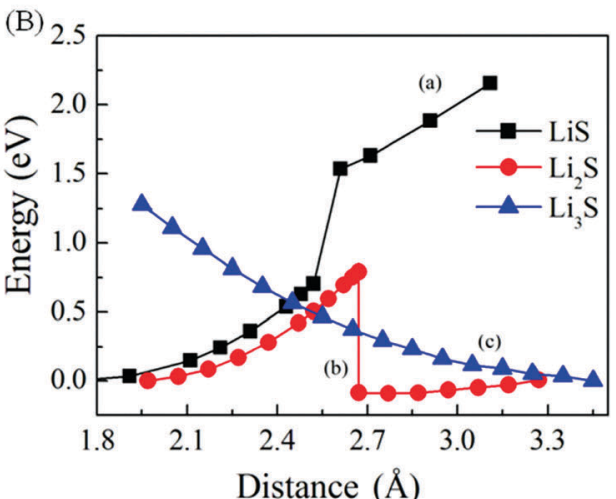

Fig. 3 Schematic representations of the dynamical process of S-desorption at the edge of double-vacancy graphene with single Li adsorption (a, LiS), double Li ion adsorption $\left(\mathrm{b}, \mathrm{Li}_{2} \mathrm{~S}\right)$ and triple $\mathrm{Li}$ ion adsorption $(\mathrm{c}, \mathrm{Li} 3 \mathrm{~S})(\mathrm{A})$ and the potential-energy curves of S-desorption in three cases $(\mathrm{B})$. Note that the direction of $\mathrm{S}$ diffusion is perpendicular to the graphene sheet. For $\mathrm{LiS}$ and $\mathrm{Li}_{2} \mathrm{~S}$, the zero points of energies are cited at the positions where $\mathrm{S}$ is trapped at the edge of doublevacancy with the adsorption of single- $\mathrm{Li}$ and double $\mathrm{Li}_{\text {. For }} \mathrm{LiS}_{3}$, the zero of energy is cited at the position where $\mathrm{S}$ is far way from the graphene plane.

treated using the nudged elastic band method and the diffusion barriers along the pathway were obtained. For $\mathrm{Li}_{2} \mathrm{~S}$, the barrier is very high - approximately $14.5 \mathrm{eV}$ if the lattice sites near the pathway are not relaxed in the diffusion of one of the Li ions in $\mathrm{Li}_{2} \mathrm{~S}$ and $3.5 \mathrm{eV}$ with relaxation. This implies that it will not be easy for $\mathrm{Li}$ to go through the graphene plane in the case of $\mathrm{Li}_{2} \mathrm{~S}$. Interestingly, in the case of $\mathrm{Li}_{3} \mathrm{~S}$, the barriers for $\mathrm{Li}$ through the double-vacancy are lower - approximately $12.7 \mathrm{eV}$ and $2.6 \mathrm{eV}$ without and with the relaxation of the lattice sites near vacancy, respectively. This relatively small barrier compared with that in the case of $\mathrm{Li}_{2} \mathrm{~S}$ can be possibly ascribed to the weakening of $\mathrm{S}$-graphene interaction by the formation of $\mathrm{Li}_{2} \mathrm{~S}$ clusters. In the case of $\mathrm{Li}_{2} \mathrm{~S}$, the diffusion of $\mathrm{Li}$ results in LiS near doublevacancy. As shown in Fig. 3, there is a strong interaction between $\mathrm{S}$ and the edge of vacancy for LiS near double-vacancy. This may be the main reason that there is a relatively large barrier for the diffusion of $\mathrm{Li}$ in the $\mathrm{Li}_{2} \mathrm{~S}$ near graphene plane. Compared to the diffusion barrier (about $0.54 \mathrm{eV}$ ) without the effect of $\mathrm{S}$ in the previous work, ${ }^{52}$ the higher barrier $(2.6 \mathrm{eV})$ is due to the attraction of $\mathrm{Li}_{2} \mathrm{~S}$ to the diffusing $\mathrm{Li}$, as illustrated in the inset of Fig. 4 . The energy will presumably decrease by following the increase of the Li number in the $\mathrm{Li}_{n} \mathrm{~S}$ cluster. On the other side, the effect of poly-sulfur (such as $\mathrm{S}_{8}$ and $\mathrm{S}_{2}$ ) on the diffusion of Li is relatively weak, since the interaction between poly-sulfur and graphene is weak, as discussed above. These results imply that the diffusion of Li through the double-vacancy is relatively facile, though there is an energy barrier in the presence of sulfur.

It is also possible that Li-ions diffuse along the plane of graphene and then go through the graphene encapsulation layer by the large pore openings freely. Here the effect of S-adsorption on the diffusion of Li around the double-vacancy is analyzed. As shown in Fig. 5, the diffusion of Li-ions near double-vacancy under the interaction of $\mathrm{S}$, LiS and $\mathrm{LiS}_{2}$ is simulated. For the case of LiS, $\mathrm{S}$ is coupled strongly to the edge of double-vacancy with the formation of chemical bonds, and the interaction between $\mathrm{Li}$ and $\mathrm{S}$ is weak. Therefore, the diffusion of Li-ions near double-vacancy with the effect of S is similar to that of Li-ions near single-vacancy, ${ }^{52}$ and there is a barrier of about $0.52 \mathrm{eV}$ due to the trapping of double-vacancy with S. For $\mathrm{Li}_{2} \mathrm{~S}$, Li makes the interaction between $\mathrm{S}$ and the edge weak, while the interaction between the diffused $\mathrm{Li}$ and $\mathrm{S}$ is increased and the barrier of Li diffusion becomes $0.68 \mathrm{eV}$ due to the 
(A)

(a)

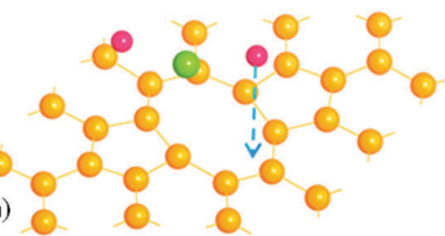

(b)

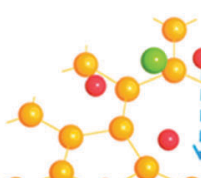

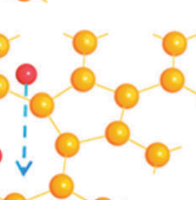

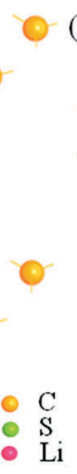

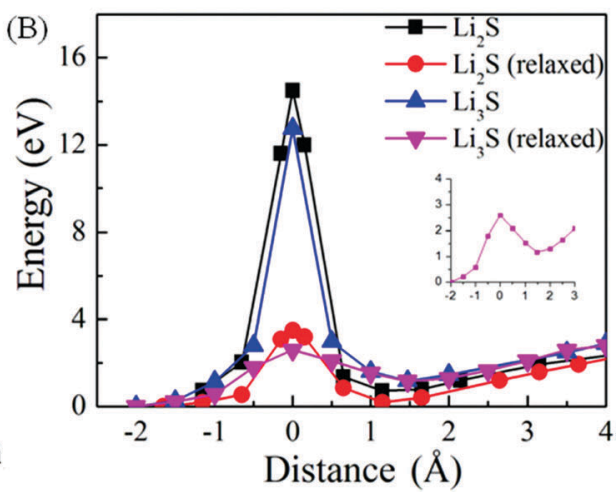

Fig. 4 Schematic representations of the diffusion of $\mathrm{Li}$ through the graphene plane by double-vacancy in the case of $\mathrm{Li}_{2} \mathrm{~S}$ and $\mathrm{Li}_{3} \mathrm{~S}(\mathrm{~A})$, and potentialenergy curves of $\mathrm{S}$ diffusion in the direction perpendicular to the graphene plane for both cases (B). Note that there is a large barrier in the process of $\mathrm{Li}$ diffusion if the lattice sites near the diffusion path are not relaxed and potential-energy curves of the case of $\mathrm{Li}_{3} \mathrm{~S}$ with the relaxation of lattice sites near the diffusion path is shown with a small energy scale in the inset of (B).

trapping effect of $\mathrm{S}$. For $\mathrm{Li}_{3} \mathrm{~S}$, the effect of the trapping of $\mathrm{S}$ becomes stronger and a deep well with a barrier of $1.71 \mathrm{eV}$ is formed. In addition, from the $\mathrm{H}_{1}$ to $\mathrm{H}_{2}$ site, an obvious barrier of about $0.18 \mathrm{eV}$ is found, compared with that of $\mathrm{LiS}$ and $\mathrm{Li}_{2} \mathrm{~S}$. From the above analysis, the diffusion of $\mathrm{Li}$ in the graphene plane is easier than that by passing through the double-vacancy.

\section{Electronic properties of pristine and double-vacancy graphene with $\mathrm{Li}_{n} \mathrm{~S}$ clusters}

To analyze the nature of interaction between the $\mathrm{Li}_{n} \mathrm{~S}$ cluster and graphene, we compute the distribution of charge in real place. In Fig. 6, the charge redistribution is analyzed based on the following formula:

$$
\Delta \mathrm{CH}(r)=\mathrm{CH}_{\mathrm{Li}-\mathrm{S}-\mathrm{g}}(r)-\mathrm{CH}_{\mathrm{Li}-\mathrm{S}}(r)-\mathrm{CH}_{\mathrm{g}}(r)
$$

where $\mathrm{CH}_{\mathrm{Li}-\mathrm{S}-\mathrm{g}}(r), \mathrm{CH}_{\mathrm{Li}-\mathrm{S}}(r)$ and $\mathrm{CH}_{\mathrm{g}}(r)$ are the real-space electronic charge distributions of the $\mathrm{Li}_{n} \mathrm{~S}$ cluster adsorbed on graphene (pristine or with double-vacancy), the isolated $\mathrm{Li}_{n} \mathrm{~S}$ cluster and isolated graphene, respectively. For pristine graphene with the absorption of $\mathrm{Li}_{2} \mathrm{~S}$ and $\mathrm{Li}_{3} \mathrm{~S}$ in Fig. $6 \mathrm{~A}$ and $\mathrm{B}$, the charge is transferred from $\mathrm{Li}_{n} \mathrm{~S}$ to the graphene plane. The charge is mostly distributed in the region under the cluster as may be anticipated. This is characteristic of the typical ionic bond. The charge from $\mathrm{Li}_{n} \mathrm{~S}$ enters graphene and occupies the bands associated with graphene. The attractive Coulomb interaction provides the binding of the cluster to graphene. With the increase of Li-ions, even the charge is

\section{(A)}

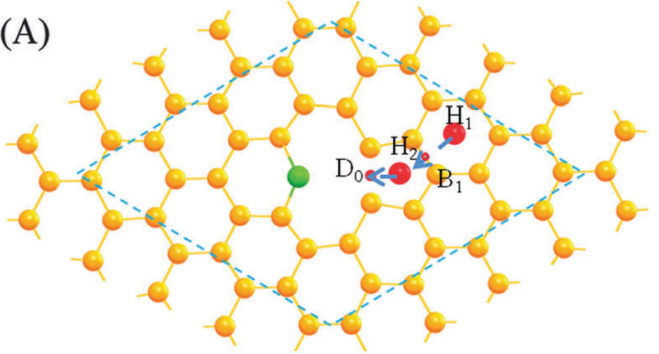

(C)

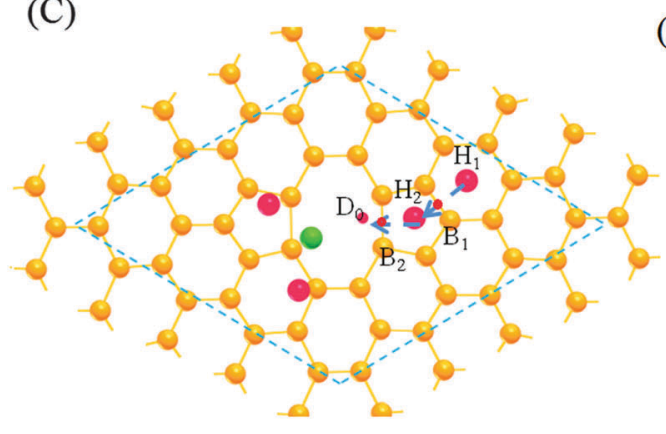

(B)

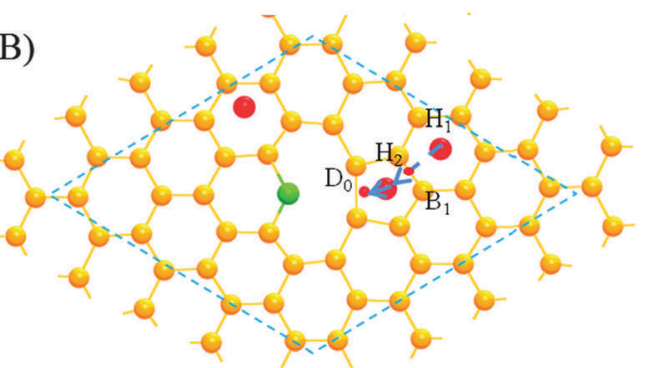

(D)

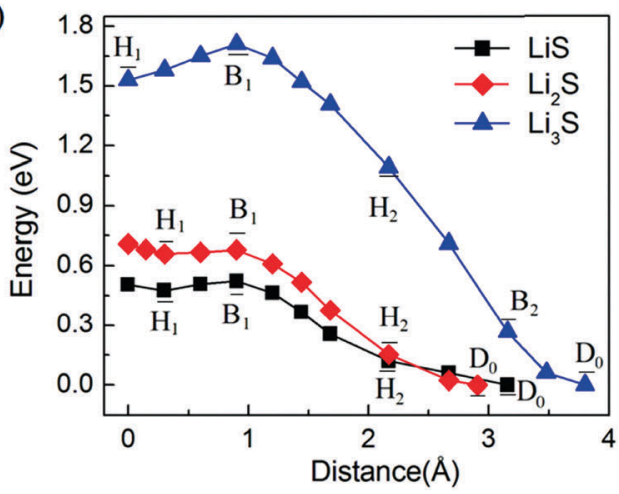

Fig. 5 Schematic representations of the paths (A, B, and C) and potential-energy curves (D) of Li diffusion on double-vacancy graphene for LiS, LiS 2 and LiS 3 . 
(A)
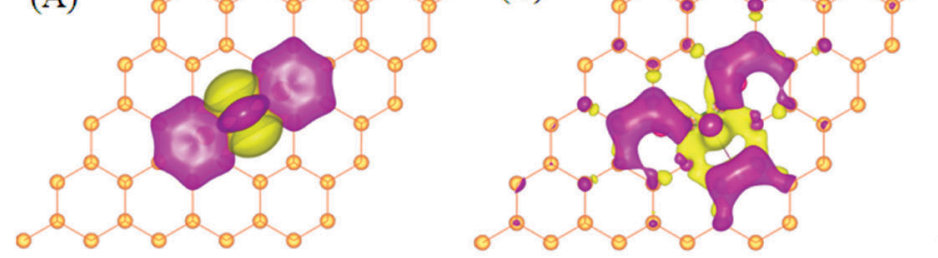

(C)

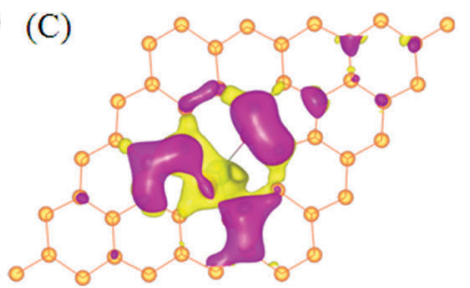

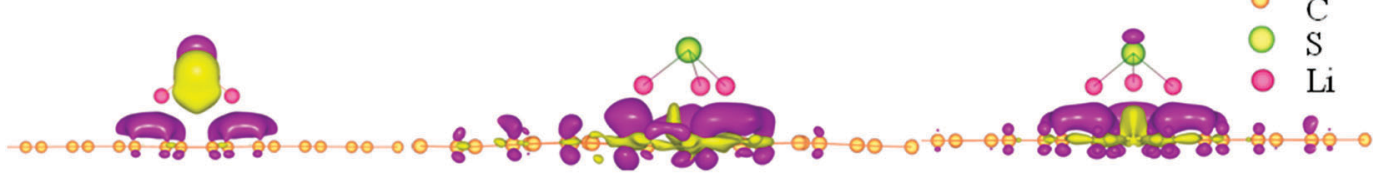

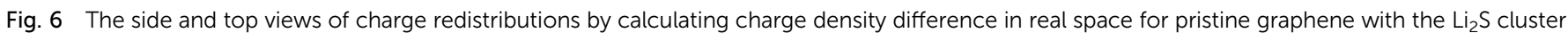

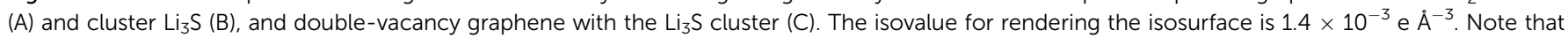
magenta and red correspond to charge accumulation and charge depletion, respectively.

transferred to the region on graphene, which is away from the cluster of $\mathrm{Li}_{n} \mathrm{~S}$ (Fig. 6B). The same case appears in doublevacancy graphene in Fig. 6C.

In graphene, the bands near the Fermi level are formed from the $\mathrm{p}_{z}$ orbitals by following $\mathrm{sp}^{2}$ hybridization. The valence and conduction bands intersect at the Fermi level with linear dispersion. When $\mathrm{S}$ is adsorbed, $\mathrm{S}-\mathrm{C}$ bonds form, which results in local breaking of $\mathrm{sp}^{2}$ hybridization. Due to the local symmetry breaking of potential, the band gap at $K$ point is opened (see Fig. S11 of ESI $\dagger$ ). The coupling between S_p and C_p $z$ results in a bonding state at about $-1.1 \mathrm{eV}$ and an antibonding state at about $1.0 \mathrm{eV}$.

When Li is introduced, the $\operatorname{Li}_{n} \mathrm{~S}$ cluster can form and the interaction between graphene and $\mathrm{Li}_{n} \mathrm{~S}$ is mainly associated with charge transfer, as above. From the band structures in
Fig. 7A and B, it is found clearly that the Fermi level is shifted up into the conduction band of graphene with spin-polarization of localized states from the $\mathrm{Li}_{n} \mathrm{~S}$ cluster (in Fig. $8 \mathrm{~A}$ ) for the cases of $\mathrm{LiS}$ and $\mathrm{Li}_{2} \mathrm{~S}$. Upon increasing the number of $\mathrm{Li}$ atoms, more electrons transfer into the conduction band of graphene. Interestingly, for the $\mathrm{Li}_{3} \mathrm{~S}$ cluster (in Fig. $7 \mathrm{C}$ ), spin-polarization disappears, and the Fermi level is shifted up to about $1 \mathrm{eV}$ above the Dirac point with the localized states from the $\mathrm{Li}_{3} \mathrm{~S}$ cluster near $-1.5 \mathrm{eV}$ (in Fig. 8A).

The introduction of double-vacancies results in the redistribution of local charge and leads to a gap in the valance band under the Dirac point in Fig. 7D. This gap is an artifact of the periodicity of the supercell, but nonetheless, indicates a likelihood of localization of states near in energy to the band crossing (Fig. 8B). At the
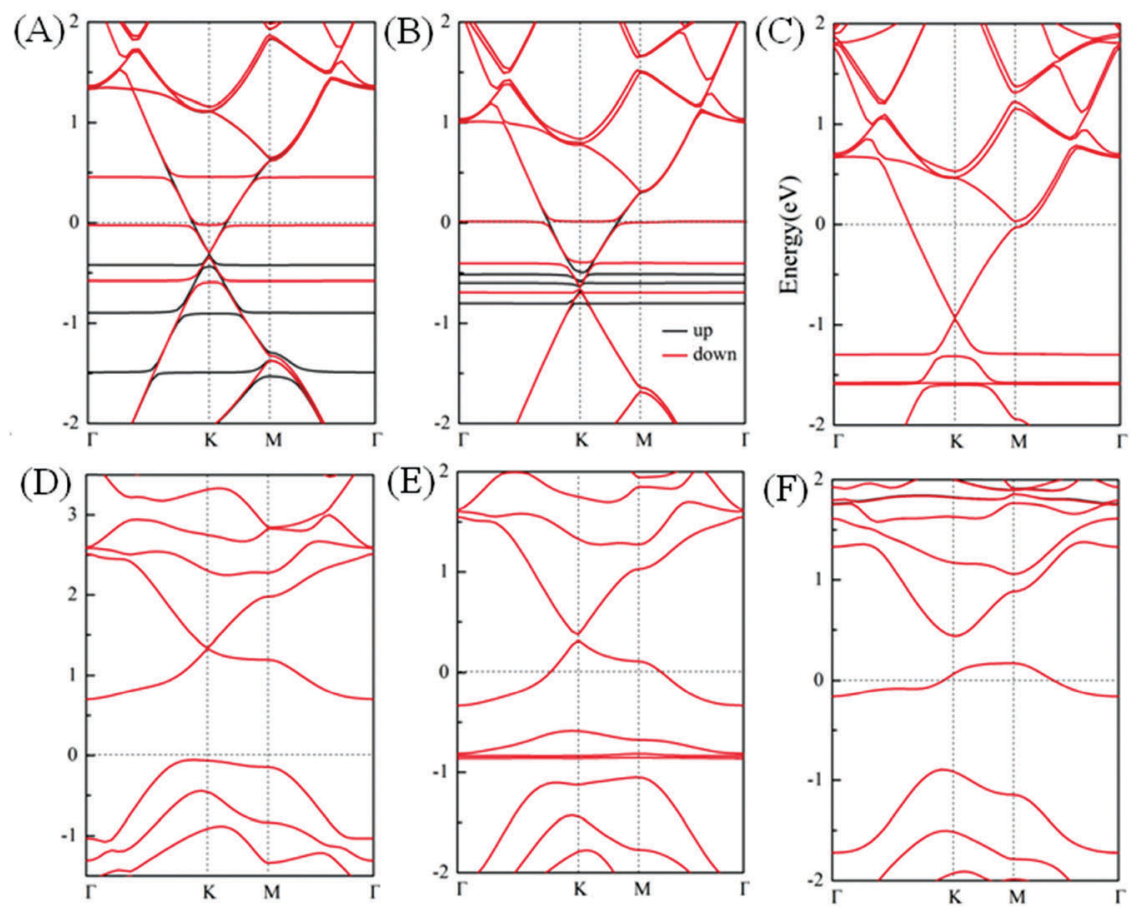

Fig. 7 Spin-polarized band structures of LiS-adsorbed graphene (A), Li $2 \mathrm{~S}$-adsorbed graphene (B), Li $\mathrm{i}_{3} \mathrm{~S}$-adsorbed graphene (C), two-vacancy graphene (D), two-vacancy graphene with adsorption of the $\mathrm{Li}_{3} \mathrm{~S}$ cluster $(\mathrm{E})$, two-vacancy graphene with one $\mathrm{S}$ adsorbed at the edge of vacancy and one $\mathrm{Li}$ adsorbed near the vacancy (LiS) (F). Note that red and black correspond to spin-up bands and spin-down bands, respectively. 

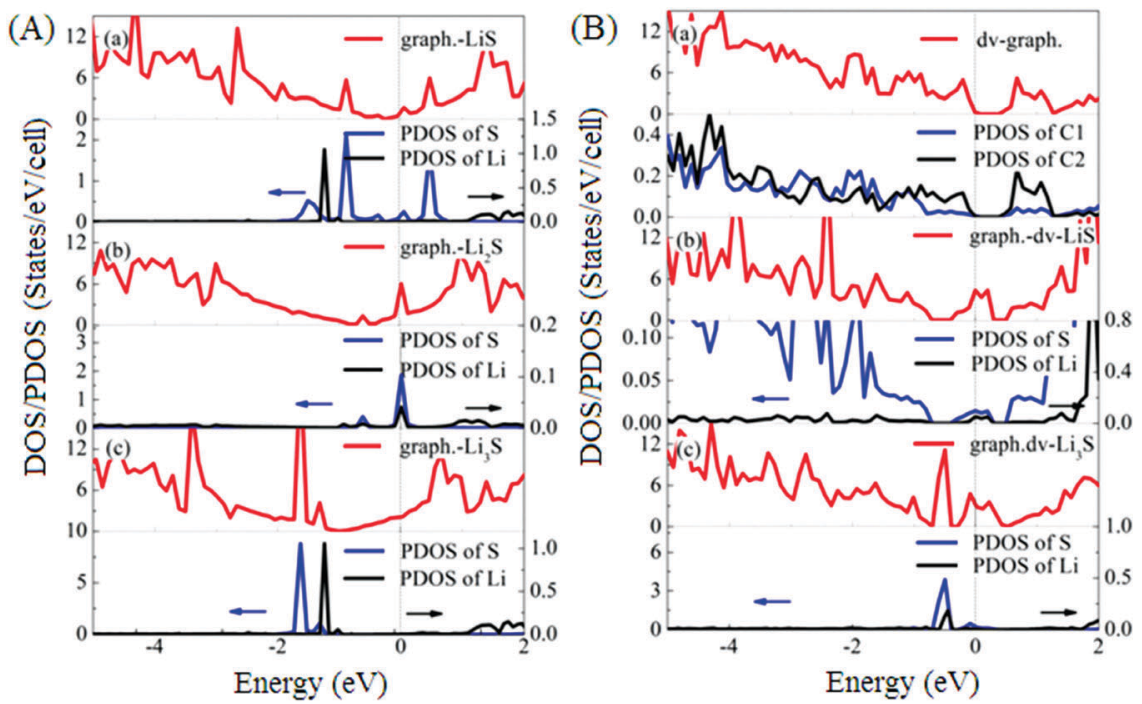

Fig. 8 The density of states (DOS) and partial density of states (PDOS) of LiS-adsorbed, $\mathrm{Li}_{2} \mathrm{~S}$-adsorbed and $\mathrm{Li}_{3} \mathrm{~S}$-adsorbed graphene (A), and doublevacancy graphene, two-vacancy graphene with one S adsorbed at the edge of vacancy and one Li adsorbed near the vacancy, and two-vacancy graphene with the adsorption of the $\mathrm{Li}_{3} \mathrm{~S}$ cluster (B). Note the PDOS of double-vacancy graphene in (B), $\mathrm{C} 2$ and $\mathrm{C} 1$, represent the carbon atom at the edge of double-vacancy and that away from double-vacancy. The DOS (PDOS) is the sum of spin-up and spin-down channels.

double-vacancy, the interaction between $\mathrm{S}$ and graphene is not weakened by a single Li in the case of LiS. As seen in Fig. 8B and Fig. S12 (ESI $\dagger$ ), it is clear that S is hybridized fully with the nearneighbor $\mathrm{C}$ from the partial density of states. A band gap is opened near the Dirac point because the interaction from the $\mathrm{S}$ atom and the Fermi level is shifted up due to the electronic doping of $\mathrm{Li}$, as seen in Fig. 7F. However, the Fermi level still is lower than the Dirac point. This implies that $\mathrm{S}$ adsorption with one $\mathrm{Li}$ atom results in the p-type doping of graphene. Considering $\mathrm{Li}_{3} \mathrm{~S}$, the formation of the $\mathrm{Li}_{3} \mathrm{~S}$ clusters breaks the S-graphene bond so that the interaction between $\mathrm{Li}_{3} \mathrm{~S}$ and graphene is just by charge transfer. The Fermi level is shifted up further relative to Lis but still is under the Dirac point, i.e. with weaker, but still p-type doping.

\section{Conclusions}

The microscopic mechanisms of interaction between $\mathrm{Li}_{n} \mathrm{~S}$ and graphene related to $\mathrm{Li}-\mathrm{S}$ batteries are explored using firstprinciples methods. It is found that the introduction of $\mathrm{Li}$ on graphene results in the increase in the distance of $\mathrm{S}$ adsorbed on graphene and the formation of $\mathrm{Li}_{n} \mathrm{~S}$ clusters. A single Li atom can lift the $\mathrm{S}$ atom with an energy barrier of $0.34 \mathrm{eV}$ and two $\mathrm{Li}$ ions can induce the desorption of $\mathrm{S}$ readily. For the double-vacancy defected graphene, the binding of $\mathrm{S}$ is stronger and at least two $\mathrm{Li}$ atoms are needed for the desorption of S at the edge of double-vacancy. It is found that the interaction between $\mathrm{Li}_{n} \mathrm{~S}$ clusters and graphene is strong due to charge transfer. This makes graphene a good encapsulation layer for preventing the dissolution of lithium polysulfide into electrolytes. However, the formation of $\mathrm{Li}_{n} \mathrm{~S}$ and adsorption of $\mathrm{S}$ at the edge of double-vacancy also limit the diffusion of $\mathrm{Li}$ into the electrolyte. This implies the importance of vacancy defects on graphene. It is found that the diffusion barrier is too high for Li to go through the graphene plane at a double-vacancy if $\mathrm{S}$ is adsorbed at the edge of double-vacancy. Interestingly, $\mathrm{S}$ can be desorbed at the edge with the trapping of Li ions near the vacancy. $\mathrm{Li}$ atoms from $\mathrm{Li}_{n} \mathrm{~S}$ can go through the graphene plane at double-vacancy with a small diffusion barrier. The analysis of electronic properties indicates that the pristine and defected graphene can have good conductivity in the process of intercalating in or/and out graphene. This is because $\mathrm{Li}_{n} \mathrm{~S}$ clusters act as dopants moving the Fermi level away from the gap position where states may be localized by defects. Understanding the interaction between lithium polysulfide and graphene and the diffusion of Li is very important since recent experimental results show that defected graphene is a good encapsulation layer for trapping $\mathrm{Li}_{n} \mathrm{~S}$ and hindering the dissolution of lithium polysulfide into the electrolyte with good electronic conductivity. The present results suggest further work clarifying the nature of defects in graphene layers in cathodes and $\mathrm{Li}_{n} \mathrm{~S}$ cluster formation in relation to atomic and electronic transport in the cathode.

\section{Acknowledgements}

The research was supported by the National Natural Science Foundation of China (Grant no. 11504123 and no. 51627805) and the National Key Research and Development Program of China (2016YFA0200401). Work at the University of Missouri was supported by the Department of Energy, BES through the MAGICS center.

\section{References}

1 P. G. Bruce, S. A. Freunberger, L. J. Hardwick and J.-M. Tarascon, $\mathrm{Li}^{-} \mathrm{O}_{2}$ and Li-S Batteries with High Energy Storage, Nat. Mater., 2012, 11, 19-29.

2 A. Luntz, Beyond Lithium Ion Batteries, J. Phys. Chem. Lett., 2015, 6, 300-301. 
3 C. M. Hayner, X. Zhao and H. H. Kung, Materials for Rechargeable Lithium-Ion Batteries, Annu. Rev. Chem. Biomol. Eng., 2012, 3, 445-471.

4 G. Zhou, S. Pei, L. Li, D. W. Wang, S. Wang, K. Huang, L. C. Yin, F. Li and H. M. Cheng, A Graphene-Pure-Sulfur Sandwich Structure for Ultrafast, Long-Life Lithium-Sulfur Batteries, Adv. Mater., 2014, 26, 625-631.

5 H. A. Salem, G. Babu, C. V. Rao and L. M. R. Arava, Electrocatalytic Polysulfide Traps for Controlling Redox Shuttle Process of Li-S Batteries, J. Am. Chem. Soc., 2015, 137, 11542-11545.

6 M. S. Islam and C. A. J. Fisher, Lithium and Sodium Battery Cathode Materials: Computational Insights into Voltage, Diffusion and Nanostructural Properties, Chem. Soc. Rev., 2014, 43, 185-204.

7 J. B. Goodenough and K.-S. Park, The Li-Ion Rechargeable Battery: A Perspective, J. Am. Chem. Soc., 2013, 135, 1167-1176.

8 Q. Zhang, Y. Wang, Z. W. Seh, Z. Fu, R. Zhang and Y. Cui, Understanding the Anchoring Effect of Two-Dimensional Layered Materials for Lithium-Sulfur Batteries, Nano Lett., 2015, 15, 3780-3786.

9 M. S. Whittingham, Lithium Batteries and Cathode Materials, Chem. Rev., 2004, 104, 4271-4302.

10 H. Wei, J. Ma, B. Li, Y. Zuo and D. Xia, Enhanced Cycle Performance of Lithium-Sulfur Batteries Using a Separator Modified with a PVDF-C Layer, ACS Appl. Mater. Interfaces, 2014, 6, 20276-20281.

11 F. Wu, J. Qian, R. Chen, J. Lu, L. Li, H. Wu, J. Chen, T. Zhao, Y. Ye and K. Amine, An Effective Approach to Protect Lithium Anode and Improve Cycle Performance for $\mathrm{Li}-\mathrm{S}$ Batteries, ACS Appl. Mater. Interfaces, 2014, 6, 15542-15549.

$12 \mathrm{X}$. Ji and L. F. Nazar, Advances in Li-S Batteries, J. Mater. Chem., 2010, 20, 9821-9826.

13 J. Liu, W. Li, L. Duan, X. Li, L. Ji, Z. Geng, K. Huang, L. Lu, L. Zhou, Z. Liu, W. Chen, L. Liu, S. Feng and Y. Zhang, A Graphene-like Oxygenated Carbon Nitride Material for Improved Cycle-Life Lithium/Sulfur Batteries, Nano Lett., 2015, 15, 5137-5142.

14 X. Niu, X. Wang, D. Wang, Y. Li, Y. Zhang, Y. Zhang, T. Yang, T. Yu and J. Tu, Metal Hydroxide - a New Stabilizer for the Construction of Sulfur/Carbon Composites as High-Performance Cathode Materials for Lithium-Sulfur Batteries, J. Mater. Chem. A, 2015, 3, 17106-17112.

15 L. Sun, W. Kong, Y. Jiang, H. Wu, K. Jiang, J. Wang and S. Fan, Super-Aligned Carbon Nanotube/Graphene Hybrid Materials as a Framework for Sulfur Cathodes in High Performance Lithium Sulfur Batteries, J. Mater. Chem. A, 2015, 3, 5305-5312.

16 J. H. Kim, K. Fu, J. Choi, K. Kil, J. Kim, X. Han, L. Hu and U. Paik, Encapsulation of S/SWNT with PANI Web for Enhanced Rate and Cycle Performance in Lithium Sulfur Batteries, Sci. Rep., 2015, 5, 8946.

17 S. Wei, L. Ma, K. E. Hendrickson, Z. Tu and L. A. Archer, Metal-Sulfur Battery Cathodes Based on PAN-Sulfur Composites, J. Am. Chem. Soc., 2015, 137, 12143-12152.

18 D.-W. Wang, Q. Zeng, G. Zhou, L. Yin, F. Li, H.-M. Cheng, I. R. Gentle and G. Q. Max Lu, Carbon-Sulfur Composites for Li-S Batteries: Status and Prospects, J. Mater. Chem. A, 2013, 1, 9382-9394.

19 H. Kim, J. Lee, H. Ahn, O. Kim and M. Park, Synthesis of Three-Dimensionally Interconnected Sulfur-Rich Polymers for Cathode Materials of High-Rate Lithium-Sulfur Batteries, Nat. Commun., 2015, 6, 7278.

20 F. Wu, J. T. Lee, F. Fan, N. Nitta, H. Kim, T. Zhu and G. Yushin, A Hierarchical Particle-Shell Architecture for Long-Term Cycle Stability of $\mathrm{Li}_{2} \mathrm{~S}$ Cathodes, Adv. Mater., 2015, 27, 5579-5586.

21 H. Chen, C. Wang, W. Dong, W. Lu, Z. Du and L. Chen, Monodispersed Sulfur Nanoparticles for Lithium-Sulfur Batteries with Theoretical Performance, Nano Lett., 2015, 15, 798-802.

22 A. Manthiram, Y. Fu, S.-H. Chung, C. $\mathrm{Zu}$ and Y.-S. Su, Rechargeable Lithium-Sulfur Batteries, Chem. Rev., 2014, 114, 11751-11787.

23 Z. Li, Y. Jiang, L. Yuan, Z. Yi, C. Wu, Y. Liu, P. Strasser and Y. Huang, A Highly Ordered Meso@Microporous CarbonSupported Sulfur@Smaller Sulfur Core-Shell Structured Cathode for Li-S Batteries, ACS Nano, 2014, 8, 9295-9303.

24 L. Pauling, On the Stability of the $S_{8}$ Molecule and the Structure of Fibrous Sulfur, Proc. Natl. Acad. Sci. U. S. A., 1949, 35, 495-499.

25 H. J. Bernstein and J. Powling, The Vibrational Spectra and Structure of Inorganic Molecules. II. Sulfur $\mathrm{S}_{8}$, Sulfur Chloride $\mathrm{S}_{2} \mathrm{Cl}_{2}$, Phosphorous $\mathrm{P}_{4}$, J. Chem. Phys., 1950, 18, 1018.

26 B. E. Warren and J. T. Burwell, The Structure of Rhombic Sulphur, J. Chem. Phys., 1935, 3, 6.

27 S. C. Abrahams, The Crystal and Molecular Structure of Orthorhombic Sulfur, Acta Crystallogr., 1955, 8, 661-671.

28 R. Xu, I. Belharouak, X. Zhang, R. Chamoun, C. Yu, Y. Ren, A. Nie, R. Shahbazian-Yassar, J. Lu, J. C. M. Li and K. Amine, Insight into Sulfur Reactions in Li-S Batteries, ACS Appl. Mater. Interfaces, 2014, 6, 21938-21945.

29 J.-J. Chen, R.-M. Yuan, J.-M. Feng, Q. Zhang, J.-X. Huang, G. Fu, M.-S. Zheng, B. Ren and Q.-F. Dong, Conductive Lewis Base Matrix to Recover the Missing Link of $\mathrm{Li}_{2} \mathrm{~S}_{8}$ during the Sulfur Redox Cycle in Li-S Battery, Chem. Mater., 2015, 27, 2048-2055.

30 Q. Qu, T. Gao, H. Zheng, Y. Wang, X. Li, X. Li, J. Chen, Y. Han, J. Shao and H. Zheng, Strong Surface-Bound Sulfur in Conductive $\mathrm{MoO}_{2}$ Matrix for Enhancing Li-S Battery Performance, Adv. Mater. Interfaces, 2015, 2, 1500048.

31 K. Ding, Y. Bu, Q. Liu, T. Li, K. Meng and Y. Wang, TernaryLayered Nitrogen-Doped Graphene/Sulfur/Polyaniline Nanoarchitecture for the High-Performance of Lithium-Sulfur Batteries, J. Mater. Chem. A, 2015, 3, 8022-8027.

32 Z. Yuan, H.-J. Peng, J.-Q. Huang, X.-Y. Liu, D.-W. Wang, X.-B. Cheng and Q. Zhang, Hierarchical Free-Standing Carbon-Nanotube Paper Electrodes with Ultrahigh SulfurLoading for Lithium-Sulfur Batteries, Adv. Funct. Mater., 2014, 24, 6105-6112.

33 Z. Zhang, H.-K. Jing, S. Liu, G.-R. Li and X.-P. Gao, Encapsulating Sulfur into a Hybrid Porous Carbon/CNT Substrate as a Cathode for Lithium-Sulfur Batteries, J. Mater. Chem. A, 2015, 3, 6827-6834. 
34 G. He, C. J. Hart, X. Liang, A. Garsuch and L. F. Nazar, Stable Cycling of a Scalable Graphene-Encapsulated Nanocomposite for Lithium-Sulfur Batteries, ACS Appl. Mater. Interfaces, 2014, 6, 10917-10923.

35 G. M. Yang, H. Z. Zhang, X. F. Fan and W. T. Zheng, Density Functional Theory Calculations for the Quantum Capacitance Performance of Graphene-Based Electrode Material, J. Phys. Chem. C, 2015, 119, 6464-6470.

36 H. Wang, Y. Yang, Y. Liang, J. T. Robinson, Y. Li, A. Jackson, Y. Cui and H. Dai, Graphene-Wrapped Sulfur Particles as a Rechargeable Lithium-Sulfur Battery Cathode Material with High Capacity and Cycling Stability, Nano Lett., 2011, 11(7), 2644-2647.

37 S. Lu, Y. Cheng, X. Wu and J. Liu, Significantly Improved Long-Cycle Stability in High-Rate Li-S Batteries Enabled by Coaxial Graphene Wrapping over Sulfur-Coated Carbon Nanofibers, Nano Lett., 2013, 13, 2485-2489.

38 S. Wu, R. Ge, M. Lu, R. Xu and Z. Zhang, Graphene-based Nano-materials for Lithium-Sulfur Battery and Sodium-ion Battery, Nano Energy, 2015, 15, 379-405.

39 F. Li, Y. Su and J. Zhao, Shuttle inhibition by chemical adsorption of lithium polysulfides in B and $\mathrm{N}$ co-doped graphene for Li-S batteries, Phys. Chem. Chem. Phys., 2016, 18, 25241-25248.

40 P. Hohenberg and W. Kohn, Inhomogeneous Electron Gas, Phys. Rev., 1964, 136, B864.

41 G. Kresse and J. Furthmüller, Efficient Iterative Schemes for $A b$ Initio Total-energy Calculations Using a Plane-Wave Basis Set, Phys. Rev. B: Condens. Matter Mater. Phys., 1996, 54, 11169-11189.

42 G. Kresse and J. Furthmüller, Efficiency of $A b$ Initio Total Energy Calculations for Metals and Semiconductors Using a Plane-Wave Basis Set, Comput. Mater. Sci., 1996, 6, 15-50.
43 J. P. Perdew, K. Burke and M. Ernzerhof, Generalized Gradient Approximation Made Simple, Phys. Rev. Lett., 1996, 77, 3865-3868.

44 S. Grimme, Semiempirical GGA-type Density Functional Constructed with a Long-range Dispersion Correction, J. Comput. Chem., 2006, 27, 1787.

45 X. F. Fan, W. T. Zheng, V. Chihaia, Z. X. Shen and J.-L. Kuo, Interaction between Graphene and the Surface of $\mathrm{SiO}_{2}$, J. Phys.: Condens. Matter, 2012, 24, 305004.

46 G. Mercurio, E. R. McNellis, I. Martin, S. Hagen, F. Leyssner, S. Soubatch, J. Meyer, M. Wolf, P. Tegeder, F. S. Tautz and K. Reuter, Structure and Energetics of Azobenzene on $\mathrm{Ag}(111)$ : Benchmarking Semiempirical Dispersion Correction Approaches, Phys. Rev. Lett., 2010, 104, 036102.

47 X. Fan, W. T. Zheng, J.-L. Kuo and D. J. Singh, Adsorption of Single $\mathrm{Li}$ and the Formation of Small Li Clusters on Graphene for the Anode of Lithium-Ion Batteries, ACS Appl. Mater. Interfaces, 2013, 5, 7793-7797.

48 K. T. Chan, J. B. Neaton and M. L. Cohen, First-Principles Study of Metal Adatom Adsorption on Graphene, Phys. Rev. B: Condens. Matter Mater. Phys., 2008, 77, 235430.

49 B. Meyer, Solid Allotropes of Sulfur, Chem. Rev., 1964, 64, 429-451.

50 B. Meyer, Elemental Sulfur, Chem. Rev., 1976, 76, 367-388.

51 T. O. Wehling, A. I. Lichtenstein and M. I. Katsnelson, Transition-Metal Adatoms on Graphene: Influence of Local Coulomb Interactions on Chemical Bonding and Magnetic Moments, Phys. Rev. B: Condens. Matter Mater. Phys., 2011, 84, 235110.

52 X. Fan, W. T. Zheng and J.-L. Kuo, Adsorption and Diffusion of Li on Pristine and Defective Graphene, ACS Appl. Mater. Interfaces, 2012, 4, 2432-2438. 